\title{
Aprendizaje del Lean Manufacturing mediante Minecraft: aplicación a la herramienta ${ }_{5} \mathrm{~S}$
}

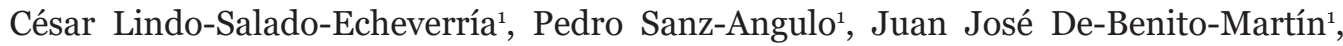 \\ Jesús Galindo-Melero ${ }^{1}$ \\ cesar.lindo@alumnos.uva.es, \{psangulo,debenito\}@eii.uva.es, jesus.galindo@uva.es \\ ${ }^{1}$ Universidad de Valladolid, Escuela de Ingenierías Industriales, Paseo del Cauce 59, 47011, Valladolid, España.
}

DOI: 10.17013/risti.16.60-75

Resumen: El Lean Manufacturing es una filosofía de producción que ha resultado ser una pieza clave para asegurar la supervivencia de numerosas empresas. Por eso, los ingenieros del futuro han de conocer y trabajar las herramientas y técnicas propias del lean a fin de que puedan utilizarlas en su actividad profesional de la forma más eficiente. Para lograrlo, además de contar con talleres formativos, es necesario emplear otros instrumentos docentes basados en las nuevas tecnologías, dado su carácter motivador y sus amplias y conocidas ventajas. En este sentido, la gamificación y, en particular, el uso de los videojuegos, constituye un enfoque innovador y atractivo. Partiendo de esta idea, en el presente documento se describe un ejemplo de aplicación del videojuego Minecraft para favorecer el aprendizaje de la metodología ${ }_{5} \mathrm{~S}$, una herramienta esencial en el éxito del Lean Manufacturing.

Palabras-clave: Aprendizaje; Gamificación; Minecraft; Lean Manufacturing; 5S.

\section{Lean Manufacturing Learning by Minecraft: application to the ${ }_{5} S$ tool}

Abstract: Lean Manufacturing is a production philosophy that has proven to be a key piece to ensure the survival of many companies. Future engineers have to learn to use the Lean tools in order to develop their professional activity more efficiently. To achieve this, in addition to training workshops, it is necessary to use teaching tools based on new technologies, given their motivating character and its widely known advantages; in this regard, the use of videogames is an innovative and attractive approach. Starting from this idea, this paper describes an application example of how we have used the videogame Minecraft to favor learning of $5 \mathrm{~S}$ methodology.

Keywords: Learning; Gamification; Minecraft; Lean Manufacturing; 5s. 


\section{Introducción}

A lo largo de los últimos años, varios profesores del Departamento de Organización de Empresas de la Universidad de Valladolid (UVa) venimos desarrollando un proceso de innovación docente y mejora continua dirigido a facilitar el aprendizaje de los contenidos relacionados con la ingeniería de organización industrial (gestión de empresas, producción, logística, economía, dirección de proyectos, recursos humanos, ...) y el desarrollo de competencias transversales que los alumnos precisan tanto a nivel profesional como personal.

Fruto de este proceso de innovación se han desarrollado y aplicado diferentes herramientas, softwares, metodologías, dinámicas de grupo, ..., que complementan todo el camino formativo realizado por el alumnado de grado y máster. Todas ellas responden a la necesidad de reorientar la labor docente hacia un modelo que promueva el binomio "conocimientos-competencias", acorde con el cambio que demanda el mercado y que, además, viene impulsando el EEES. Se busca que los alumnos adquieran los conocimientos y el saber propio de su profesión (el qué enseñar) mientras cultivan las competencias clave (ligadas al cómo enseñar) para el desempeño de su actividad profesional (Sanz, De Benito, \& Galindo, 2011).

Además, han sido construidas de forma que sean fáciles de usar, atractivas y cercanas, con un doble propósito: por un lado, el de motivar a los alumnos e involucrarles en el proceso formativo (buscamos convertirles en elementos activos y responsables de su propio aprendizaje) y, por otro, favorecer que puedan ser utilizadas por otros docentes o profesionales, tanto del área de ingeniería de organización como de cualquier otro ámbito de conocimiento.

Algunos de estos desarrollos se enmarcan dentro de la actividad de diferentes proyectos de innovación docente (PID), como es el caso de los PID centrados en el desarrollo y mejora de la metodología SACC (Sanz et al., 2011) o el proyecto más reciente, "Escuela Lean: estudio y desarrollo de diversas líneas de mejora orientadas a apoyar la labor docente y el aprendizaje de los alumnos dentro del marco del EEES”, en el que se circunscribe el trabajo que aquí presentamos.

El Lean Manufacturing representa una forma de entender la actividad empresarial que se ha revelado a nivel mundial como referente a la hora de configurar una organización capaz de mejorar sus procesos y resultados de forma continua. Por este motivo, Renault Consulting y la Universidad de Valladolid inauguraron en 2014, en la Escuela de Ingenierías Industriales de la UVa, la primera Escuela de aprendizaje Lean en España (http://escuela-lean.es/). En ella, se trata de enseñar, de forma práctica, cómo eliminar de los procesos (productivos, logísticos, etc.) todo aquello que no aporta valor para, de este modo, ganar en competitividad.

Sin duda alguna, el uso de la Escuela Lean ha permitido mejorar el aprendizaje de esta filosofía en asignaturas de diferentes grados y másteres de ingeniería que se están impartiendo en la UVa. Sin embargo, consideramos que existen todavía multitud de formas de transmitir y trabajar los conceptos y herramientas propios del Lean en dichas titulaciones, mientras se trabajan competencias y habilidades que nuestros alumnos requerirán tanto a nivel personal como profesional. 
Por este motivo, en el proyecto de innovación docente mencionado anteriormente se ha trabajado en diferentes líneas de actuación, entre las que se encuentran: el desarrollo de nuevos productos para incorporar al taller Lean (lo que incluye el diseño de nuevas piezas, sus procesos productivos, sus especificaciones de calidad,...), modelado y experimentación de diferentes configuraciones de la Escuela Lean (fabricación por lotes, one-piece-flow,...) con la herramienta de simulación Witness, aplicación de la metodología de aprendizaje SACC (http://metodologiaaprendizajesacc.blogs.uva. es/),..., y la creación de modelos Lean en MineCraft.

Precisamente, en este documento presentamos un ejemplo de entorno Lean creado con el juego Minecraft. En concreto, se describe un modelo desarrollado para explicar y abordar la metodología $5 \mathrm{~S}$, uno de los principios básicos de la filosofía Lean. Pero antes, y a fin de lograr una mejor compresión del trabajo realizado, abordaremos brevemente los conceptos clave del trabajo, como son: gamificación, Minecraft, Lean Manufacturing y las $5 \mathrm{~S}$.

\section{Gamificación y Minecraft}

En los últimos años hemos asistido a un gran desarrollo tecnológico que ha cambiado la forma en la que nos comunicamos, nos relacionamos, trabajamos, ..., y, por supuesto, el modo en que disfrutamos. En este sentido, las nuevas plataformas, tanto hardware como software, han posibilitado el uso de los videojuegos en diferentes áreas de conocimiento.

Pero a medida que aumenta su empleo, también lo hace el interés por descubrir las razones que hacen de los videojuegos un medio tan eficaz para motivar y para desarrollar competencias y valores tan importantes como la creatividad, la participación, el esfuerzo, el comportamiento proactivo, el trabajo en equipo, ..., tan necesarias en el día a día de cualquier persona.

No es de extrañar, por tanto, que multitud de investigadores hayan comenzado a estudiar la forma de emplear los videojuegos en contextos no lúdicos a fin de aprovechar sus ventajas y puntos fuertes. Surge así el concepto de gamificación o ludificación, que hace referencia a la tendencia actual de emplear los videojuegos, o mecánicas inspiradas en ellos, en entornos y aplicaciones tan dispares como la salud, comunicación, producción, servicios, ..., y, en especial, la educación.

La gamificación constituye un enfoque especialmente fértil en contextos como la educación y la empresa (Pérez, 2012). En los últimos años han surgido diferentes experiencias de aplicación del aprendizaje basado en juegos a escenarios de formación reglada, particularmente en el entorno de la educación superior (Poy-Castro, MendanaCuervo, \& Gonzalez, 2015). Uno de los juegos que más potencial tiene es Minecraft (https://minecraft.net/), un videojuego de tipo mundo abierto o sandbox, que permite construir cualquier entidad que el usuario se imagine mediante cubos con texturas tridimensionales.

Es fácil encontrar ejemplos de utilización de Minecraft en el ámbito docente (Sáez \& Domínguez, 2014; Schifter \& Cipollone, 2013; Martínez, Del Cerro, \& Morales, 2014;...), fundamentalmente en estudios de primaria y secundaria, e incluso existen escuelas que están incorporando este juego al currículum escolar; por ejemplo, la escuela Viktor 
Rydberg, en Suecia, ha incorporado una materia obligatoria exclusivamente dedicada a Minecraft (Holloway, 2013) en la que los estudiantes construyen, gestionan y planifican a través de este juego.

Si bien Minecraft no ofrece grandes gráficos, es muy fácil de manejar y permite hacer y construir prácticamente todo lo que uno quiera. En este sentido, como en este trabajo vamos a simular procesos productivos, va a ser una herramienta tremendamente útil ya que nos permite hacerlo de una forma visual. También va a permitir "simular" el movimiento de los elementos y el paso del tiempo.

Los bloques son las unidades básicas estructurales en Minecraft. Se encargan de dar forma al entorno del juego y pueden ser recogidos y utilizados por el jugador. Para transformar los bloques en objetos más complejos utilizaremos principalmente dos procesos: la fabricación y la fundición. La mayor parte de los objetos, bloques y herramientas de Minecraft los fabricaremos utilizando una mesa de trabajo, mientras que algunos productos más refinados se obtienen mediante la fundición en hornos. Por otro lado, aunque menos importante, también podemos generar "pociones" o mezclas utilizando mesas específicas.

Para controlar, moldear y automatizar los procesos hemos empleado un material especial, llamado redstone o piedra rojiza. Este material hace posible la creación de "circuitos de redstone" en los que se pueden incluir interruptores, botones, palancas, placas de presión y puertas lógicas en combinación con puertas, dinamita y/o raíles de vagonetas (Redstone, 2015).

\section{Lean Manufacturing, la Escuela Lean y las $5 \mathrm{~S}$}

El comportamiento de los mercados actuales está presidido por las exigencias de los clientes, que buscan satisfacer su demanda con productos cada vez más personalizados. En este contexto, cualquier empresa se ve en la obligación de fabricar lo que demanda el cliente justo en el momento oportuno, en la cantidad y calidad que precisa y a un precio competitivo. Se necesitan, por tanto, medios productivos suficientemente flexibles que permitan a las empresas responder a esta demanda con nuevos productos, o variaciones de los ya existentes, utilizando los recursos disponibles de un modo eficiente (Gil, Sanz, De Benito, \& Galindo, 2012).

Esta necesidad constituye el leitmotiv del Lean Manufacturing o Producción AjustadaEsbelta (Womack, Jones, \& Roos, 1992; Womack \& Jones, 2005; Cuatrecasas-Arbós, 2006, 2010) y del World Class Manufacturing o Fabricación de Clase Mundial (Schonderber, 1991, 1996; Rubrich \& Watson, 2004). Estas filosofías pretenden conseguir la excelencia en la fabricación a través de un proceso permanente de mejora continua en el que van eliminando todas aquellas tareas y recursos que no añadan valor desde el punto de vista del cliente, teniendo siempre presente el respeto por el trabajador.

Para alcanzar este objetivo genérico existe un gran número de herramientas o técnicas, que afrontan diferentes problemáticas. Por ejemplo: el SMED o Single Minute Exchange of Die, desarrollada por Shigeo Shingo (1991, 2003), permite reducir drásticamente el tiempo de preparación de un cambio de referencia; el sistema de planificación Kanban, desarrollado por Taiichi Ohno (1991), sirve para controlar la cadena logística desde un 
punto de vista de la producción siguiendo un enfoque pull; el Jidoka (Ohno, 1991), o automatización con un toque humano, permite que los procesos tengan su propio autocontrol de calidad solucionando los problemas en el momento que se producen e impidiendo que éstos se propaguen aguas abajo en la cadena productiva; etc.

Dentro de estas herramientas cabe destacar la metodología $5 \mathrm{~S}$, considerada clave en el proceso de implantación del Lean. Nacida en Toyota en los años sesenta, esta metodología tiene por finalidad obtener zonas de trabajo mejor organizadas, más ordenadas y limpias de forma duradera a fin de lograr mayores niveles de productividad, calidad, seguridad,..., y una eliminación de los desperdicios.

Dadas sus múltiples ventajas, entre las que se encuentra la obtención de resultados casi inmediatos, las $5 \mathrm{~S}$ presentan una amplia difusión a nivel mundial. Son numerosas, y muy variadas, las organizaciones que lo emplean: desde empresas industriales a empresas de servicios, pasando por centros de salud y hospitales, laboratorios, centros educativos, etc.

Las $5 \mathrm{~S}$ se basan en cinco palabras japonesas que comienzan por la letra S y que definen las fases de implantación. La primera fase es Seiri, que consiste en clasificar los elementos o herramientas de trabajo a fin de mantener en el puesto lo estrictamente necesario. La siguiente S es Seiton, en la que lo necesario debe ser ordenado e identificado para facilitar su acceso y uso.

Una vez ordenados los elementos necesarios, el área de trabajo debe limpiarse, Seiso, para mantener un alto nivel de desempeño. En la cuarta etapa, Seiketsu, se eliminan las causas de la suciedad y el desorden y se elabora un procedimiento estándar de las tres primeras S. Por último, y a fin de asegurar que todas las mejoras alcanzadas se mantengan en el tiempo y no se regrese a las prácticas anteriores, debe verificarse que los estándares se cumplen; es lo que se conoce como Shitsuke.

\section{Aprendizaje de las $5 \mathrm{~S}$ a través de Minecraft}

En este apartado se explica la implantación y funcionamiento de las 5S sobre un modelo creado en Minecraft con el que se pretende mostrar, de una manera sencilla y visual, en qué consiste cada uno de las etapas que componen esta filosofía.

Por ello, se ha diseñado un sencillo proceso productivo, con el propósito de aplicar las distintas etapas de las $5 \mathrm{~S}$ sobre él. En primer lugar, se va a describir el proceso creado para implantar esta teoría.

\subsection{Situación inicial}

Para realizar la representación visual de la aplicación de las $5 \mathrm{~S}$ se ha construido una planta donde se fabricarán tartas de calabaza. La Figura 1 muestra el esquema productivo y los materiales utilizados en la creación de dichas tartas.

Como se distingue en el esquema, primero se recolectan las cañas de azúcar y se procesan a fin de transformarlas en azúcar (Figura 2). Para ayudar en esta tarea se cuenta con una máquina que corta los tallos de las plantas de caña. Una vez recogidos los tallos hay que transformarlas en azúcar, lo que se hace con la ayuda de la mesa de trabajo. 


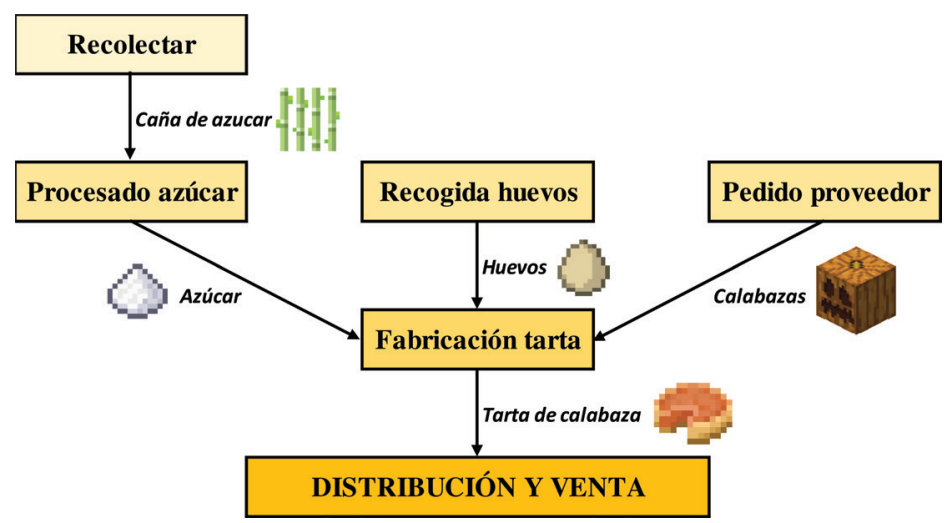

Figura 1 - Flujograma productivo del modelo $5 \mathrm{~S}$ desarrollado.

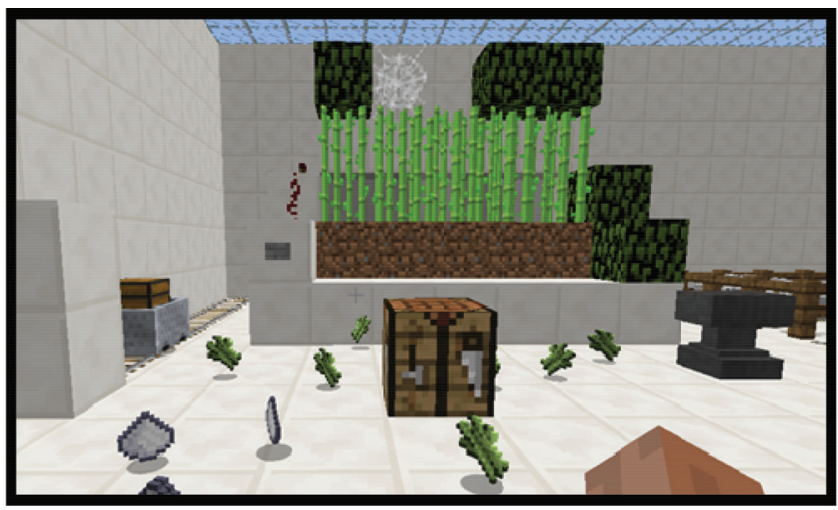

Figura 2 - Situación inicial de la zona de generación de azúcar.

El siguiente paso consiste en recoger los huevos de un pequeño "corral”, que no está automatizado, y al lado del cual se encuentra el "almacén” donde se apilan las calabazas que recibiremos de los proveedores (Figura 3).

Por último, nos encontramos con la mesa de trabajo donde se realizarán las tartas. Las carretillas cargadas con los materiales que se utilizarán en el proceso, llegarán a la pequeña plataforma que se observa en la imagen (Figura 4).

Aunque la producción se puede realizar tal y como se ha descrito (Figura 5), se puede apreciar claramente que la planta no es perfecta y dista mucho de cumplir unos mínimos de limpieza, orden o estandarización. 


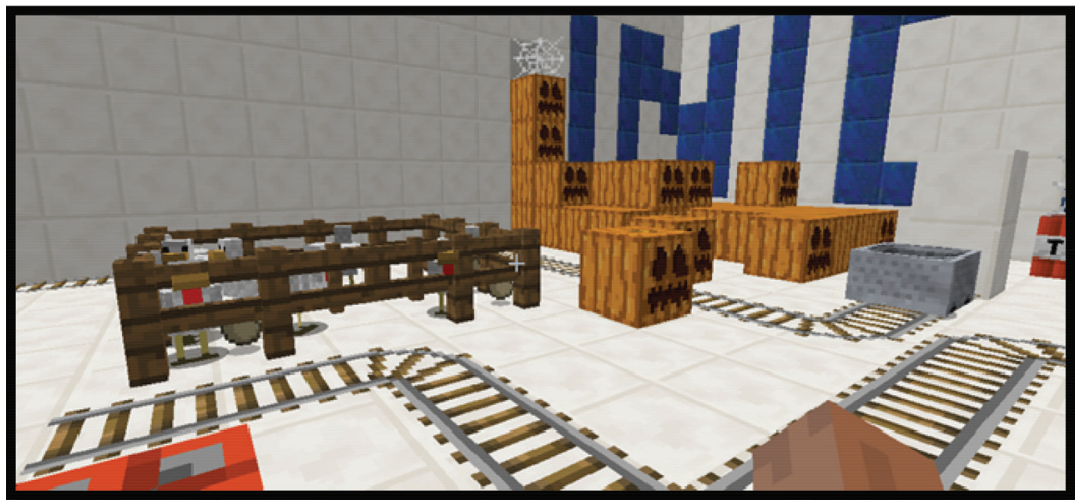

Figura 3 - Situación inicial de la zona de producción de huevos y almacenamiento de calabazas.

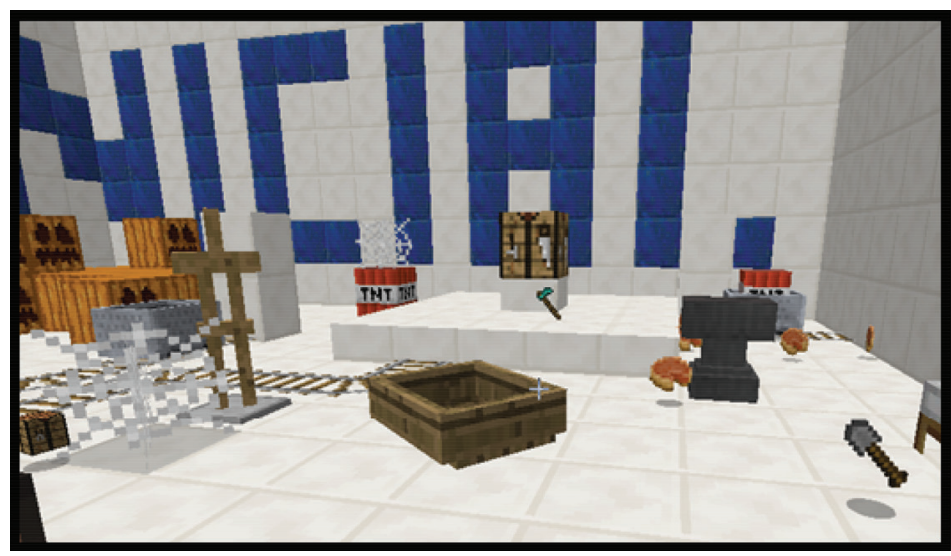

Figura 4 - Situación inicial de la zona de fabricación de la tarta de calabaza.

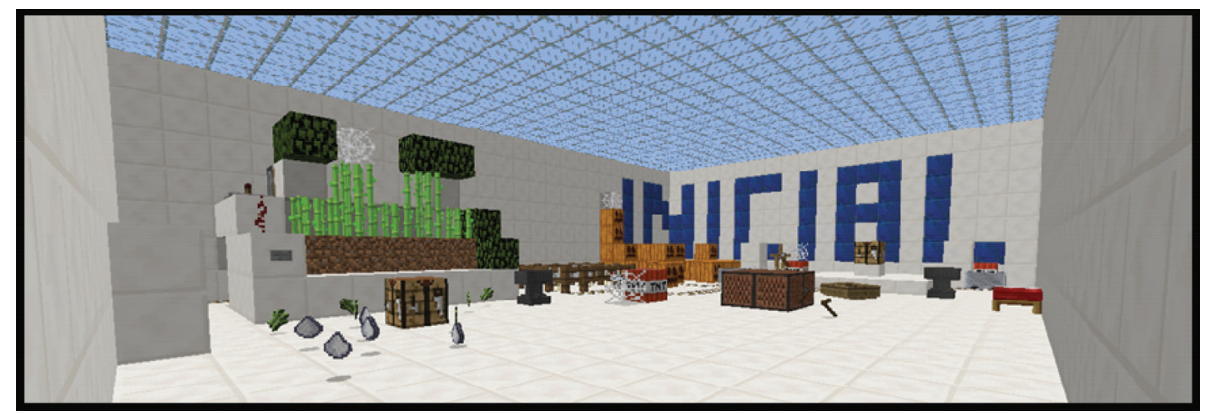

Figura 5 - Vista general que muestra la situación inicial de la planta. 


\subsection{Seiri}

La primera S es Seiri que consiste en separar lo que es necesario de lo que no lo es, pero también clasificar lo necesario por su naturaleza. De esta manera vamos a conseguir tener una planta donde únicamente encontremos los artículos y herramientas necesarias. Se van a eliminar todos los objetos que consideramos innecesarios y reubicaremos los elementos de uso poco frecuente (Figura 6).

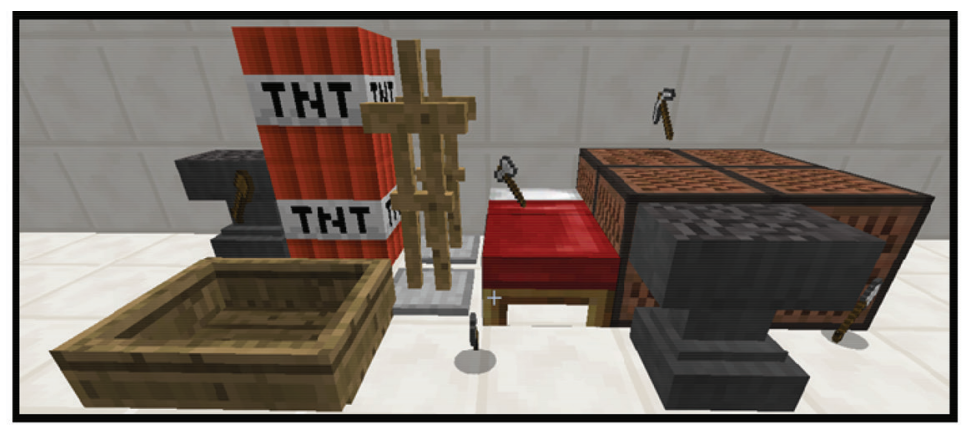

Figura 6 - Separar los objetos necesarios.

Como se observa en la imagen final (Figura 7), después de aplicar Seiri se detecta mejor de qué herramientas disponemos y con qué materiales contamos para realizar nuestras tareas. Este mejor control visual también nos asegura reducir el número de material extraviado, a controlar mejor nuestros stocks y a reducir posibles situaciones inseguras.

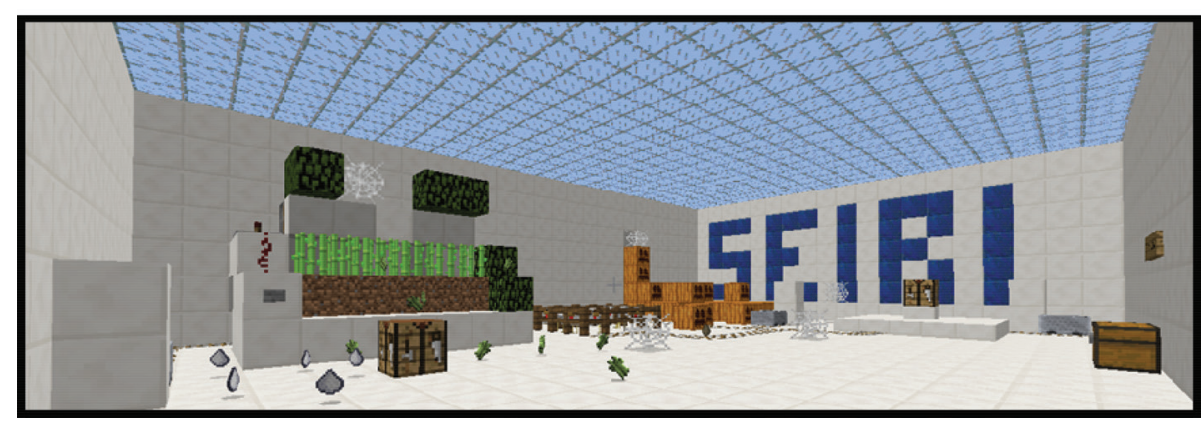

Figura 7 - Vista general de la planta tras aplicar Seiri.

\subsection{Seiton}

La frase que mejor define esta segunda $\mathrm{S}$ es el conocido principio de Henry Fayol (1916): "Un lugar para cada cosa y cada cosa en su lugar". Consiste en ordenar lo que anteriormente habíamos considerado como útil, facilitando así el acceso a los objetos más utilizados. 


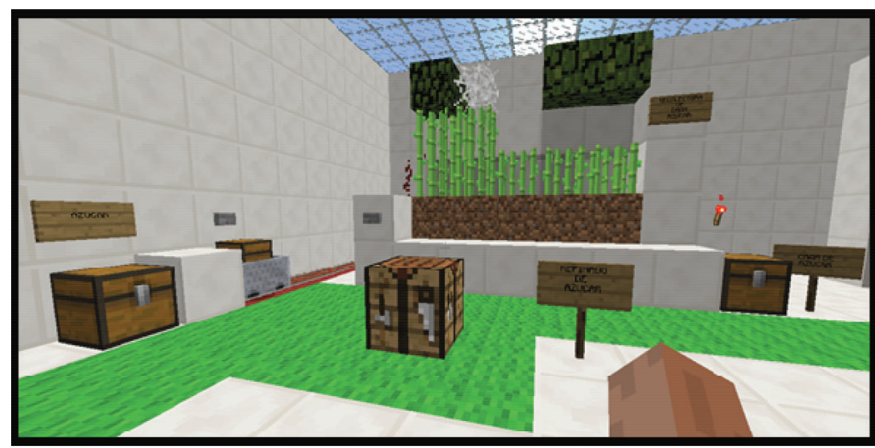

Figura 8 - Material ordenado tras la aplicación de Seiton.

Partiendo de esta idea, en la planta se ha situado cada objeto en un lugar concreto y fijo, teniendo en cuenta su naturaleza (Figura 8); de esta forma se facilita tanto la recogida de objetos, como su posterior devolución al mismo lugar. También se ha limitado el número de unidades a fin de mantener en todo momento el mismo stock de seguridad.

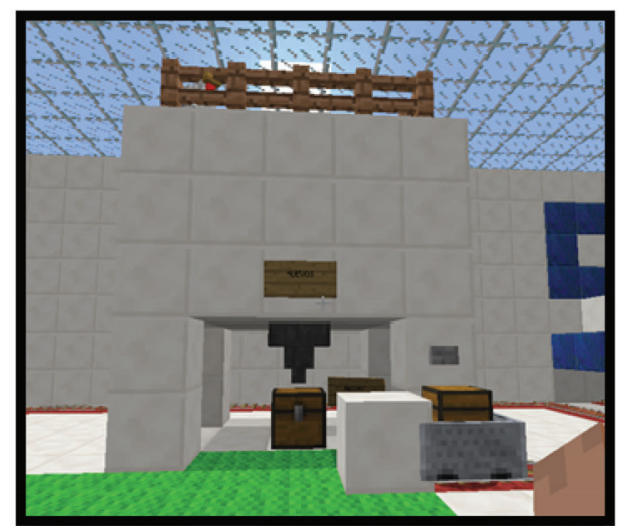

Figura 9 - Resultado de la aplicación de Seiton a la recogida de huevos.

También se ha cambiado la recogida de huevos, ya que estorbaba al paso de las carretillas; ahora se ha colocado en una zona más alta (Figura 9) a la que se tiene acceso a través de unas escaleras traseras.

En la Figura 10 se pueden apreciar otros cambios introducidos en esta fase. Por un lado, se ha delimitado el área de cada centro de trabajo o almacenamiento. También se ha utilizado un código de colores en el suelo para marcar las zonas sobre las que es seguro que anden las personas (en color verde) y la zona para carretillas (en rojo).

Además, se han automatizado los movimientos de las carretillas, de manera que vuelvan a su lugar original, una vez hayan realizado su cometido. Éste es uno de los puntos más importantes de Seiton, ya que nos va a ayudar a mantener siempre el estado inicial. 


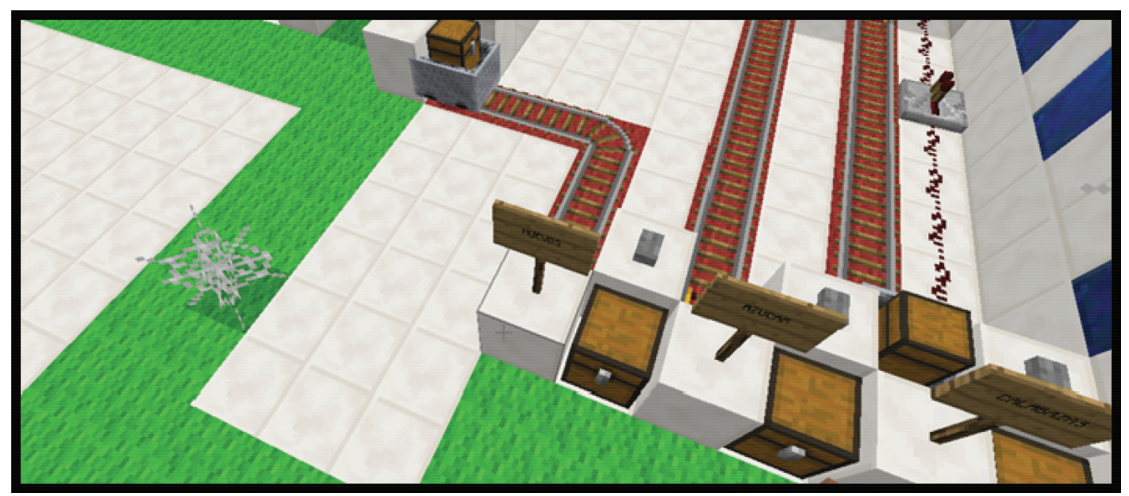

Figura 10 - Delimitación de las zonas de trabajo y desplazamiento.

La Figura 11 permite apreciar la evolución que ha sufrido la planta en tan solo dos pasos; en dicha imagen se aprecia claramente cómo se ha logrado una mejor visualización de los puestos de trabajo, ubicando cada elemento en un lugar concreto y facilitando tanto la recogida como la devolución de los objetos que se emplean en el proceso productivo.

\subsection{Seiso}

El significado de esta S refleja lo que vamos a hacer, "limpieza". Sin embargo, no sólo vamos a limpiar lo que ya está sucio, sino evitar que se ensucie de nuevo tomando medidas de higiene. Para ello, tenemos que descubrir las fuentes de suciedad de nuestra planta y solucionar el problema desde el origen, utilizando técnicas como los "cinco porqués”, diagramas de Pareto, 8D, etc. De este modo, evitaremos que aparezca nueva suciedad y desorden.

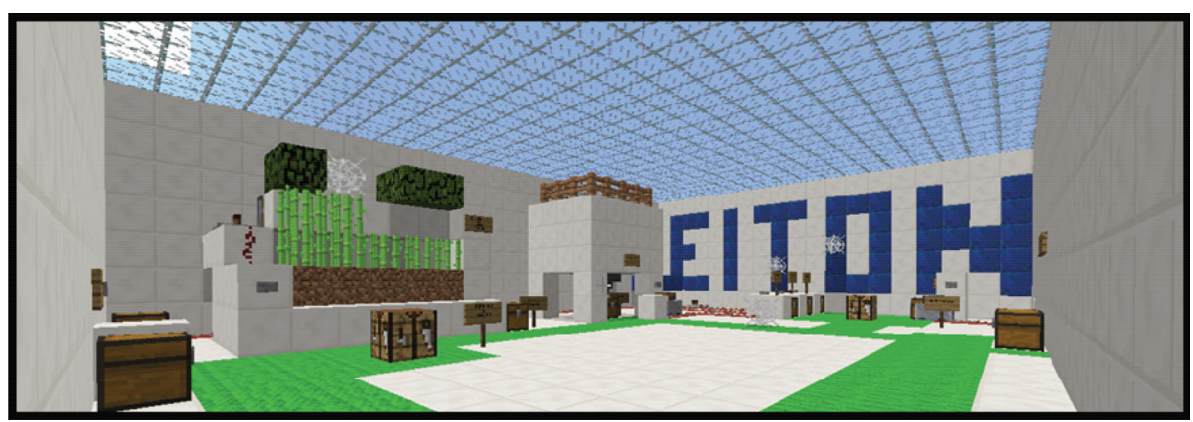

Figura 11 - Vista general de la planta tras la aplicación de Seiton.

Partiendo de esta premisa, en esta etapa primero se ha eliminado toda la suciedad de la planta y, a continuación, se han modificado los procesos que generaban dicha suciedad, a fin de lograr que no vuelva a aparecer. Así, por ejemplo, en la máquina recolectora se ha utilizado el agua (una pequeña cascada) y un acristalamiento para evitar que se distribuyan en la planta los residuos producidos por las cañas de azúcar (Figura 12). 


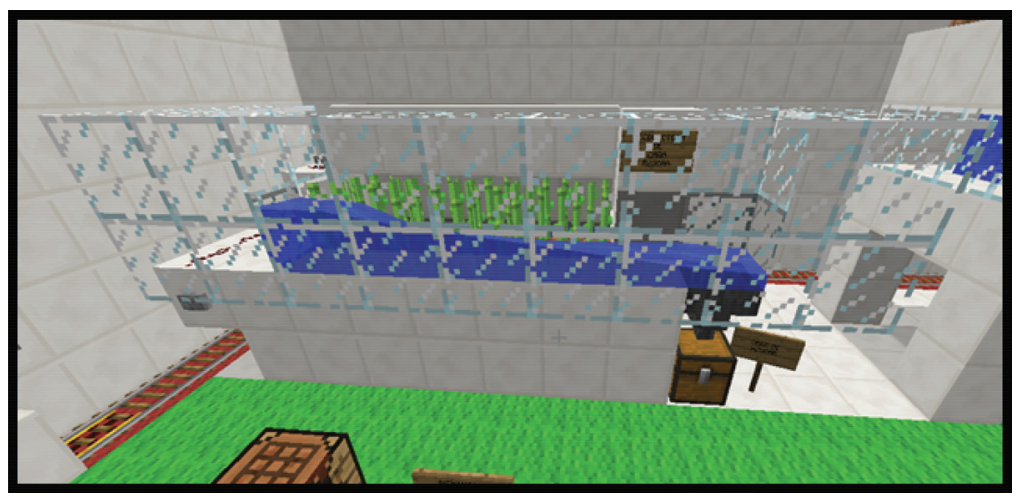

Figura 12 - Aplicación de la fase Seiso al recolector de cañas de azúcar.

En la granja de pollos se ha automatizado el proceso con un sistema similar. Como se ha hecho en las "S" anteriores, se va a mostrar una captura de la vista global (véase la Figura 13) para que se pueda observar la mejora alcanzada, en este caso, a través de la aplicación de Seiso.

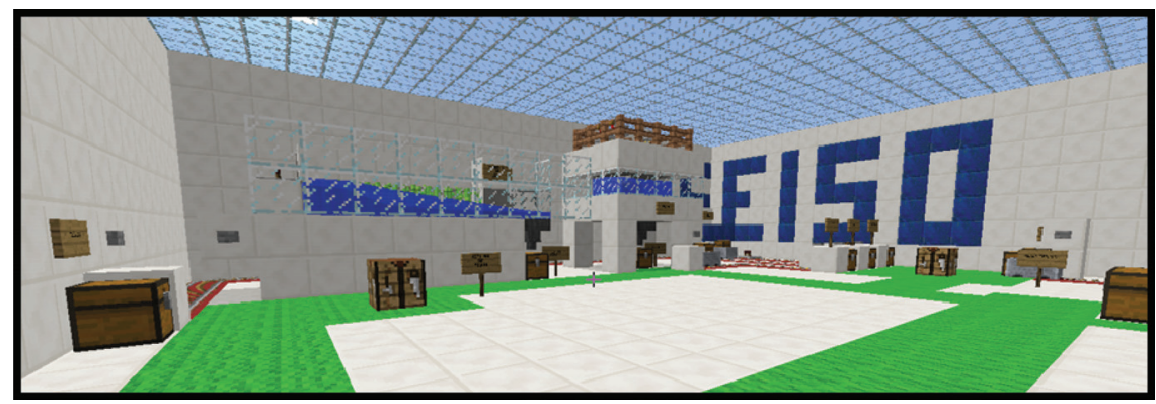

Figura 13 - Vista general de la planta tras la etapa Seiso.

Tras la aplicación de Seiso, parte de las tareas de limpieza e inspección las va a tener que realizar el propio operario, por lo que va a adquirir un mayor conocimiento del equipo que manipula. Además, conseguimos mejorar la rentabilidad de los equipos productivos y aumentar su vida útil.

\subsection{Seiketsu}

En esta etapa llevamos a cabo un proceso de estandarización que nos va a permitir mantener y conservar lo que ya hemos conseguido en las $3 \mathrm{~S}$ anteriores. Para ello, hay que implantar una serie de estándares o procedimientos, de forma que se elimine la posibilidad de volver a la situación inicial. 
En primer lugar, se ha creado un baúl junto a las herramientas de uso poco frecuente (Figura 14); en él se encuentra un libro que lleva el registro de quién utiliza esa herramienta con el objetivo de que después de utilizarse las herramientas vuelvan al lugar que les corresponde.

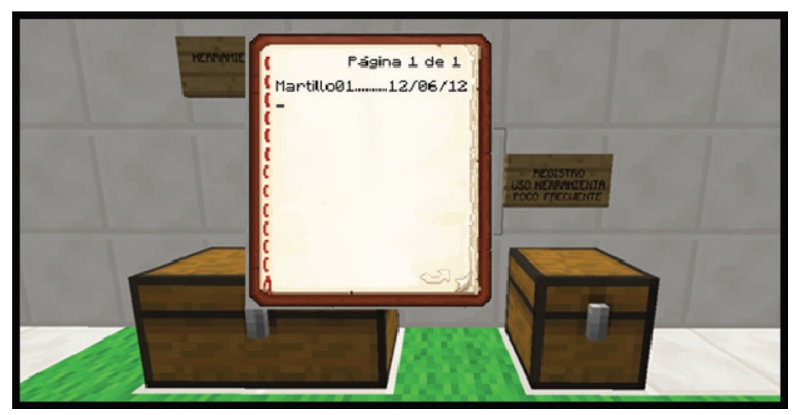

Figura 14 - Aplicación del Seiketsu a las herramientas de uso poco frecuente.

Por otro lado, en toda la planta se han instalado diferentes elementos (Figura 15): carteles informativos que describen cómo tienen que realizarse los distintos procesos; pulsadores por si se detecta algún fallo en la maquinaria; lámparas luminosas junto a cada baúl que guarde materia que se utiliza en el proceso (la luz se enciende si el stock baja del nivel de seguridad); etc.

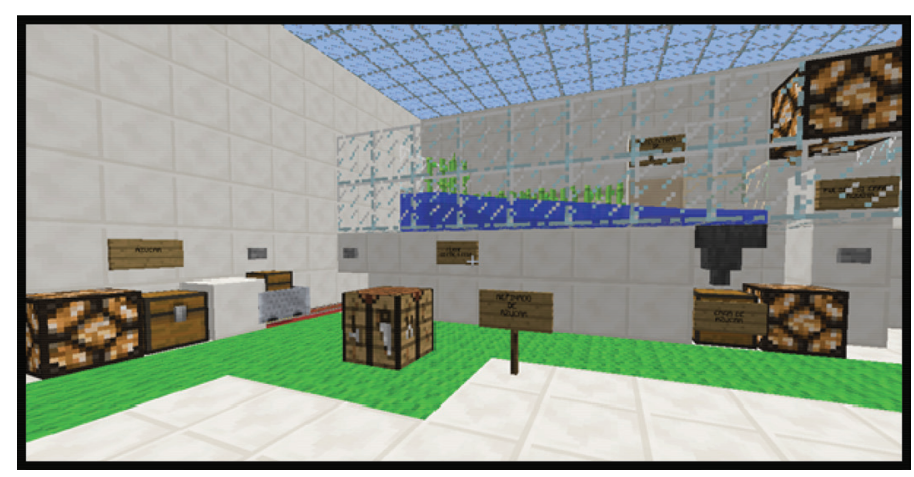

Figura 15 - Aplicación de la cuarta S a la máquina de producción de cañas de azúcar.

Cabe destacar que se ha automatizado la vuelta de los carritos a su posición original una vez los operarios hayan descargado su contenido; de esta manera, nos aseguramos que los baúles marcados como vacíos vuelven a llenarse.

Por último, la Figura 16 muestra una vista general final de cómo ha afectado la aplicación de las primeras $4 \mathrm{~S}$ sobre nuestra factoría. 


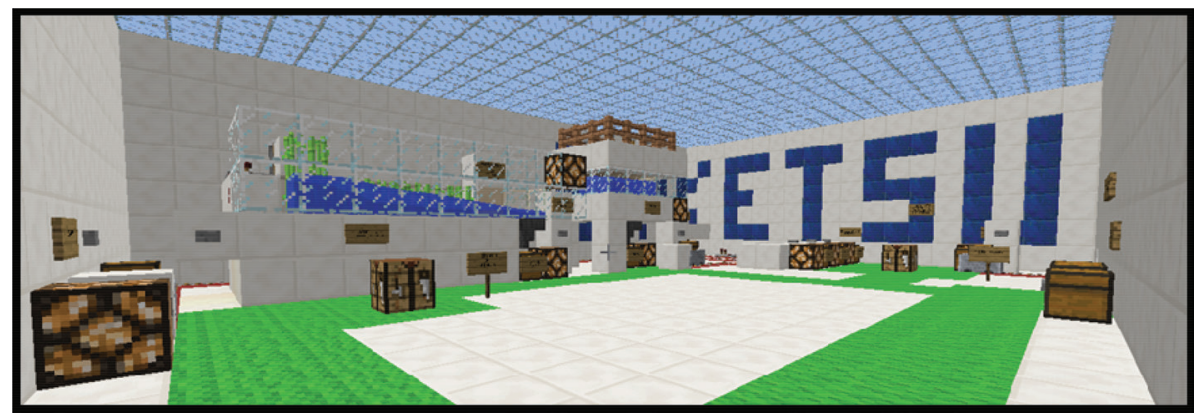

Figura 16 - Vista general de la planta tras la fase Seiketsu.

\subsection{Shitsuke}

No es posible mostrar este paso en Minecraft, ya que tiene un carácter claramente teórico. En esta etapa se refuerza lo que ya hemos realizado anteriormente, al predicar la autodisciplina para mantener lo ya logrado y al promover las auditorías.

Shitsuke está estrechamente relacionado con el término japonés Kaizen, que significa mejora en castellano. Esta filosofía no nos va a permitir retroceder en lo que ya hemos conseguido, y nos forzará a mantener una mejora continua.

Si no mantenemos esta disciplina no nos habrá servido de nada todo el esfuerzo realizado en las $4 \mathrm{~S}$ anteriores, por lo que todas las personas que forman parte de la plantilla tienen que estar mentalizadas de esto. Así concluye la implantación de las $5 \mathrm{~S}$ en esta factoría Minecraft.

\section{Conclusiones y líneas futuras}

Uno de los objetivos del proyecto de innovación docente en el que nos encontramos inmersos era conocer las posibilidades de los videojuegos, y en concreto de Minecraft, a la hora de mostrar y trabajar, de una forma muy visual y didáctica, el funcionamiento de distintas herramientas del Lean Manufacturing. Tras el trabajo realizado, podemos concluir que el uso de Minecraft para lograr este propósito ha sido un éxito, ya que nos proporciona una gran libertad a la hora de diseñar y construir diferentes procesos productivos y las plantas en las que se desarrollan.

Para apoyar esta conclusión hemos construido varios entornos productivos en Minecraft. En este artículo, en concreto, se ha mostrado cómo aplicar con éxito en este videojuego una de las herramientas clave del Lean Manufacturing como son las $5 \mathrm{~S}$; se ha seleccionado como experiencia piloto por tratarse de una metodología muy sencilla y una de las bases del éxito de la filosofía Lean. 
Para desarrollar esta metodología $5 \mathrm{~S}$ en el videojuego se ha creado, en primer lugar, un modelo de planta productiva en Minecraft, en el que se elaboran tartas de calabaza a partir de diferentes materias primas. Si bien este modelo no nos proporciona datos reales sobre su implantación, sí que nos permite hacernos una idea clara y visual de los resultados que podemos obtener tras la aplicación de la metodología.

De hecho, se puede apreciar fácilmente como la factoría simulada cambia radicalmente a medida que se van aplicando las distintas $\mathrm{S}$; se empieza con una nave caótica que evoluciona hacia una planta más eficiente, limpia y segura a medida que se avanza en el desarrollo de la metodología. De esta forma, los alumnos no sólo aprenden sobre la metodología $5 \mathrm{~S}$, sino que pueden visualizar fácilmente las mejoras que aporta su aplicación.

Por otro lado, y como se ha mostrado en el artículo, el modelo sirve para dar a conocer y trabajar otras herramientas Lean útiles como, por ejemplo, los cinco porqués, los diagramas causa-efecto de Ishikawa, etc. También sirve de base para construir nuevos entornos productivos que desarrollen otras herramientas Lean.

En este sentido, actualmente estamos trabajando en la creación de nuevos modelos Minecraft para mostrar el funcionamiento de un sistema de producción Kanban y el método Heijunka. A medida que se van afrontando nuevos conceptos y herramientas Lean es posible mejorar los procesos productivos ya creados en Minecraft, y viceversa, lo que permite ver las sinergias que se producen al emplear diferentes herramientas de forma simultánea.

Entre las líneas de actuación que surgen a partir del trabajo realizado, consideramos que Minecraft se puede combinar con otros programas que permitan realizar tareas de aprendizaje en paralelo. Por ejemplo, se podría utilizar Witness para calcular el número de tarjetas o contenedores Kanban que vamos a necesitar en nuestras factorías simuladas, o desarrollar software que permita obtener programas que nivelen no sólo la producción sino los recursos empleados.

Otra línea de actuación consiste en el uso de MinecraftEdu (http://minecraftedu. com/), la aplicación de Minecraft a la educación, cuya principal ventaja es que permite crear sesiones en las que participen un gran número de alumnos. De esta forma, podríamos conseguir que diferentes jugadores trabajasen simultáneamente, bien en horario lectivo o bien fuera de él, en una misma planta productiva, lo que facilitaría la simulación de los distintos procesos productivos.

También sería posible considerar que cada participante desarrollase su propio sistema, y que luego éstos se interconectaran entre sí. Esta posibilidad permitiría simular aspectos como la distribución o el transporte entre centros e incluso facilitaría el estudio de diferentes formas organizativas; así, podría considerarse, por ejemplo, la creación y operativa de Organizaciones Virtuales Dinámicas (Sanz-Angulo \& De-Benito-Martín, 2009). 


\section{Agradecimientos}

El trabajo que aquí se ha presentado constituye una de las líneas de innovación docente que varios profesores de la sección de Ingenierías Industriales del departamento de Organización de Empresas y Comercialización e Investigación y Comercialización de Mercados de la UVa venimos desarrollando a lo largo de los últimos años. Recientemente, esta labor ha sido reconocida por el Consejo Social de la Universidad de Valladolid en su última convocatoria de Premios de Innovación Educativa. Desde estas líneas queremos agradecer dicho reconocimiento, que supone un nuevo incentivo más para seguir innovando en el ámbito educativo y avanzar en la adaptación de la enseñanza a las necesidades actuales.

\section{Referencias}

Cuatrecasas-Arbós, L. (2006). Claves de Lean Management: un enfoque para la alta competitividad en un mundo globalizado. Barcelona: Gestión 2000, DL.

Cuatrecasas-Arbós, L. (2010). Lean management: la gestión competitiva por excelencia. Barcelona: Profit DL.

Fayol, H. (1916). Administration industrielle et générale. Bulletin de la Société de l'Industrie minérale.

Gil, M.A., Sanz, P., Benito, J.J., \& Galindo, J. (2012). Definición de una metodología para una aplicación práctica del SMED. Técnica industrial, 298, 46-54.

Holloway, J. (2013, January 14). School imposes compulsory Minecraft lessons [Blog post]. Retrieved August 20, 2015, from http://www.gizmag.com/compulsoryminecraft-lessons/25781/

Martínez, F.J., Del Cerro, F., \& Morales, G. (2014). El uso de Minecraft como herramienta de aprendizaje en la Educación Secundaria Obligatoria. En: Navarro, J., Gracia, Ma . D., Lineros, R. \& Soto, F.J. (Coords.) Claves para una educación diversa. Murcia: Consejería de Educación, Cultura y Universidades.

Ohno, T. (1991). El sistema de producción Toyota. Más allá de la producción en gran escala. Barcelona: Ediciones Gestión 2000.

Pérez, O. (2012). 'Ludificación' en la narrativa audiovisual contemporánea. TELOS, Revista de Pensamiento sobre Comunicación, Tecnología y Sociedad, 93, 1-10.

Poy-Castro, R., Mendana-Cuervo, C., \& Gonzalez, B. (2015). Diseño y evaluación de un juego serio para la formación de estudiantes universitarios en habilidades de trabajo en equipo. RISTI, Revista Ibérica de Sistemas y Tecnologías de la Información, n.spe3, 71-83. doi:10.17013/risti.e3.71-83

Redstone (n.d.). In Gamepedia, Retrieved September 10, 2015, from http://minecraftes.gamepedia.com/Redstone

Rubrich, L., \& Watson, M. (2004). Implementing World Class Manufacturing. $2^{a}$ edición. Indiana: WCM Associates. 
Sáez, J., \& Domínguez, C. (2014). Integración pedagógica de la aplicación Minecraft Edu en educación primaria: un estudio de caso. Pixel-Bit. Revista de Medios y Educación, 45, 95-110. doi: 10.12795/pixelbit.2014.i45.07

Sanz, P., de Benito, J.J., \& Galindo, J. (2011). La metodología de aprendizaje SACC. Desarrollo práctico de Competencias Transversales y Digitales en los Egresados Universitarios a través de la metodología SACC. Revista Docencia e Investigación, 21, 289-308.

Sanz-Angulo, P., \& De-Benito-Martín, J.J. (2009). Aplicación de Sistemas Inteligentes en la Creación de Organizaciones Virtuales Dinámicas para la Prevención del Maltrato Infantil. RISTI, Revista Ibérica de Sistemas y Tecnologías de la Información, 4, 69-84.

Schifter, C., \& Cipollone, M. (2013). Minecraft as a teaching tool: One case study. In R. McBride \& M. Searson (Eds.), Proceedings of Society for Information Technology \& Teacher Education International Conference 2013, 2951-2955. Chesapeake, VA: Association for the Advancement of Computing in Education (AACE).

Schonberger, R.J. (1991). Hacia la excelencia en la fabricación: lecciones aplicadas de simplificación. Madrid: Espasa-Calpe.

Schonberger, R.J. (1996). World Class Manufacturing: The Next Decade. New York: The Free Press.

Shingo, S. (2003). Una revolución en la producción: el sistema SMED. $4^{\text {a }}$ edición. Madrid: Tecnologías de Gerencia y Producción.

Womack, J.P., \& Jones, D.T. (2005). Lean Thinking: cómo utilizar el pensamiento Lean para eliminar los despilfarros y crear valor en la empresa. Barcelona: Gestión 2000.

Womack, J.P., Jones, D.T., \& Roos, D. (1992). La máquina que cambió el mundo. Madrid: MacGraw-Hill. 